\title{
Risk score for non-small cell lung cancer patients starting checkpoint inhibitor treatment
}

This article was published in the following Dove Press journal: Cancer Management and Research

\author{
Stefan Diem, ${ }^{1-3, *}$ Mirjam \\ Fässler, 2,* Omar Hasan Ali, ${ }^{2,4}$ \\ Marco Siano,' Rebekka \\ Niederer, ${ }^{2}$ Fiamma Berner, ${ }^{2}$ \\ Guillaume-Alexandre Roux, ${ }^{2}$ \\ Christoph Jakob Ackermann,' \\ Sabine Schmid, ' Sabine \\ Güsewell, ${ }^{5}$ Martin Früh, 1,6 \\ Lukas Flatz ${ }^{1,2,4,5,7}$ \\ 'Department of Oncology/ \\ Haematology, Kantonsspital St. Gallen, \\ St. Gallen, Switzerland; ${ }^{2}$ Institute \\ of Immunobiology, Kantonsspital \\ St. Gallen, St. Gallen, Switzerland; \\ ${ }^{3}$ Department of Oncology/ \\ Haematology, Spital Grabs, St. \\ Gallen, Switzerland; ${ }^{4}$ Department of \\ Dermatology, University Hospital \\ Zürich, Zürich, Switzerland; ${ }^{5}$ Clinical \\ Trials Unit, Kantonsspital St. Gallen, \\ St. Gallen, Switzerland; ${ }^{6}$ University of \\ Bern, Bern, Switzerland; ${ }^{7}$ Department \\ of Dermatology/Allergology, \\ Kantonsspital St. Gallen, St. Gallen, \\ Switzerland \\ *These authors contributed equally to \\ this work
}

Correspondence: Lukas Flatz

Department of Dermatology/

Allergology, Kantonsspital St. Gallen,

Rorschacherstrasse 95, 9007 St. Gallen,

Switzerland

Tel +4 I 7| 494 I III

Email lukas.flatz@gmail.com

Stefan Diem

Department of Oncology/Hematology,

Kantonsspital St. Gallen, Rorschacher-

strasse 95, 9007 St. Gallen, Switzerland

Tel +4I 7I 494 IIII

Fax +4I 7I 4946325

Email stefan.diem@kssg.ch
Background: Prognosis of metastatic non-small cell lung cancer significantly improved with the availability of checkpoint inhibitors (anti-PD-1/PD-L1). Unfortunately, reliable biomarkers to predict treatment benefit are lacking.

Patients and methods: We prospectively collected clinical and laboratory data of 56 non-small cell lung cancer patients treated with a checkpoint inhibitor. The aim was to identify baseline parameters correlating with worse outcome and to create a risk score that enabled to stratify patients into different risk groups. As inflammation is known to promote tumor growth, we focused on inflammation markers in the blood. Disease control (DC) was defined as complete response, partial response, and stable disease on CT scan according to RECIST 1.1.

Results: Half of the patients achieved DC. Four parameters differed significantly between the DC group and the no disease control group: Eastern Cooperative Oncology Group performance status $(P=0.009)$, number of organs with metastases $(P=0.001)$, lactate dehydrogenase $(P=0.029)$, and ferritin $(P=0.005)$. A risk score defined as the number of these parameters $(0=$ no risk factor) exceeding a threshold (Eastern Cooperative Oncology Group performance status $\geq 2$, number of organs with metastases $\geq 4$, lactate dehydrogenase $\geq 262 \mathrm{U} / \mathrm{L}$, and ferritin $\geq 241$ $\mu \mathrm{g} / \mathrm{L}$ ) was associated with overall survival and progression-free survival. Overall survival at 6 and 12 months is as follows: Scores $0-1$ : $95 \%$ and $95 \%$; Score 2: $67 \%$ and $\leq 33 \%$; Scores $3-4$ : $15 \%$ and $0 \%$. Progression-free survival at 6 and 12 months is as follows: Scores $0-1: 81 \%$ and 50\%; Score 2: $25 \%$ and $\leq 25 \%$; Scores 3-4: $0 \%$ and $0 \%$.

Conclusion: We propose an easy-to-apply risk score categorizing patients into different risk groups before treatment start with a PD-1/PD-L1 antibody.

Keywords: NSCLC, checkpoint inhibitor, biomarkers, risk score, response, survival

\section{Introduction}

Non-small cell lung cancer (NSCLC) accounts for $85 \%$ of all lung cancers. Median survival of patients with metastatic NSCLC treated with standard platinum-based chemotherapy is $\sim 12$ months. ${ }^{1}$

Since the approval of anti-programmed death-1/programmed death-ligand 1 agents (PD-1/PD-L1), novel treatment options in both adenocarcinoma and squamous cell carcinoma have become available. By blocking the inhibitory signal between PD-1 on T-cells and PD-L1 on tumor cells (checkpoints), T-cells are able to attack cancer cells leading to their apoptosis. ${ }^{2}$ Large Phase III trials showed overall response rates (ORR) between $20 \%$ and $50 \%$ with significantly increased progression-free survival (PFS) and overall survival (OS) compared with chemotherapy in the second-line 
setting and in patients with PD-L1 expression of $\geq 50 \%$ in the first-line setting, leading to the approval of nivolumab, pembrolizumab, and atezolizumab. ${ }^{2-5}$

Toxicity is manageable and rather low compared with classic chemotherapy combination strategies. Side effects are mostly autoimmune effects and can affect potentially all organs. They occur most frequently in the thyroid gland, lung, colon, and skin. However, grade 3 and 4 toxicities can be life-threatening with a fatality rate of up to $10 \% .^{2-5}$

Given these risks of potential toxicities, the high treatment costs, and importantly the fact that a significant proportion of patients do not respond to immunotherapy, reliable biomarkers are urgently needed for better patient selection and to avoid potential harm to patients unlikely to benefit. Various predictive markers have been extensively investigated including PD-L1 expression on tumor cells by immunohistochemistry. However, PD-L1 expression is still controversially discussed as patients with PD-L1 negative tumors may also show a response to therapy. Furthermore, testing for PD-L1 is not standardized and the methodology in trials is rather heterogeneous..$^{2-5}$

Another predictive biomarker that has been studied in this setting is high tumor mutational burden (TMB). ${ }^{6}$ An exploratory analysis of the Phase III checkmate 026 trial with nivolumab suggested improved ORR and PFS for patients with high TMB treated with nivolumab in the first-line setting compared with chemotherapy. ${ }^{7,8}$ In a retrospective series of patients with KRAS-mutated adenocarcinoma of the lung, mutational inactivation of STK11/LKB1 emerged as genomic predictors of de novo resistance to checkpoint blockade, ${ }^{9}$ and in melanoma patients loss of function mutations in JAK1 and JAK2 were associated with acquired resistance to checkpoint inhibitors. ${ }^{10}$ Recently, the importance of TMB as an independent biomarker was validated in a Phase III trial of nivolumab and ipilimumab in the first-line setting. ${ }^{11}$ Although TMB appears to be a promising independent biomarker, it is costly, and the definition of exact thresholds per megabase will be needed using distinct next-generation-sequencing platforms and related panels, and the minimal genome coverage required in order to maintain a high predictive value will have to be proposed.

In metastatic melanoma, several routine laboratory parameters were demonstrated to be associated with clinical outcome: lactate dehydrogenase (LDH), lymphocyte count, and eosinophil count. ${ }^{12-16}$ Markers associated with inflammation such as C-reactive protein (CRP) were shown to be a marker for tumor progression. ${ }^{17,18}$

The aim of this prospective study was 1) to identify baseline parameters associated with a worse outcome with checkpoint inhibitor treatment; 2) to analyze blood values including inflammation markers and acute phase proteins (blood sedimentation rate [BSR], fibrinogen, CRP, and ferritin); and 3) to create an easy-to-apply risk score that enables to stratify patients to different risk groups before treatment start to predict treatment outcome.

\section{Patients and methods}

We prospectively collected data of NSCLC patients treated with a PD1/PD-L1 targeting antibody in their standard dose (nivolumab $3 \mathrm{mg} / \mathrm{kg}$ every 2 weeks, pembrolizumab $2 \mathrm{mg}$ / $\mathrm{kg}$ every 3 weeks, pembrolizumab $200 \mathrm{mg}$ flat dose in the first-line setting, and atezolizumab 1,200 mg flat dose every 3 weeks). All patients were treated for metastatic disease either as a first-line treatment or as a second-line treatment after chemotherapy. In total, we included 56 patients from the Kantonsspital St. Gallen, Switzerland. All baseline blood parameters were collected at the day of treatment start. Clinical data were extracted from the electronic patient record system. Patients with concomitant infectious diseases or systemic steroids at treatment start were excluded. The response was assessed after 2 months by CT scans according to RECIST 1.1 criteria. ${ }^{19}$ We defined treatment benefit as disease control (DC) according to the following criteria: complete remission (CR), partial remission (PR), and stable disease (SD). Patients with progressive disease at the first CT scan were categorized as no disease control (NDC). CT scans were repeated according to standard local practice every 2 months.

The study was approved by the local ethics committee (Ethikkommission Ostschweiz, EKOS 16/079) and written informed consent was obtained from each participant prior to inclusion. The study was carried out in accordance with the Declaration of Helsinki principles.

\section{Statistical analyses}

Thirteen potential predictors for DC status were considered in this study. Parameters were chosen according to existing literature predominantly in melanoma patients being treated with checkpoint inhibitors. ${ }^{20}$ These included sex, Eastern Cooperative Oncology Group (ECOG) performance status at baseline, number of organs with metastases, and 10 blood parameters: BSR, CRP, LDH, ferritin, fibrinogen, leukocytes, lymphocytes, granulocytes, eosinophils, and basophils. For the number of organs with metastases, lymph nodes and soft tissue were counted as one organ irrespective of the number of sites involved.

To identify the most relevant predictors, numeric variables were plotted against DC status, and distributions were compared with Wilcoxon rank sum tests. The distribution 
of sex was compared with a chi-squared test. Only predictors with statistically significant differences between DC or NDC groups $(P<0.05)$ were included in further analyses. The significant predictors were analyzed with receiver operating characteristic (ROC) curves to determine the overall strength of association (area under the curve [AUC]), the optimal cut point for the prediction of DC (maximizing the sum of sensitivity and specificity), and the predictive values obtained with this cut point.

The cut points determined through ROC analysis were used to dichotomize each predictor, and an overall predictive score was calculated as the number of predictors with values above the cut point. Higher score values indicate a higher probability of rapid disease progression.

OS and PFS were compared between patients with and without initial DC with Kaplan-Meier curves and log-rank tests. The same was done with patients grouped by their predictive score to test whether the score also has a prognostic value for further disease progression.

\section{Results}

\section{Patient characteristics}

Fifty-six NSCLC patients who received at least one cycle of immunotherapy with a PD-1/PD-L1 antibody were included in the study. The median age was 67.6 years, $57 \%(n=32)$ were male, the majority of patients had an adenocarcinoma $(n=41$, $73 \%$ ) and received immunotherapy as a second-line treatment $(80 \%, n=45)$. Only 11 patients $(20 \%)$ received immunotherapy in the first-line setting. ECOG performance status was $0-1$ in most patients $(78 \%, n=44)$; only 12 patients with ECOG performance status $\geq 2$ were included in the analysis. KRAS mutation was found in 24 patients (43\%), and only four patients $(7 \%)$ had a targetable oncogenic driver mutation (EGFR/ALK). Information about PD-L1 expression was available for 43 patients, of which 29 had a PD-L1 expression of $\geq 1 \%$. Detailed characteristics are shown in Table 1 .

\section{Association of predictors with DC}

Of the 56 patients included, half achieved DC $(n=28,50 \%)$, 13 (23\%) with PR, and 15 (27\%) with SD at the first CT scan. Four of the baseline parameters investigated were significantly different $(P<0.05)$ between $\mathrm{DC}$ and NDC: ECOG performance status $(P=0.009)$, number of organs with metastases (Norgans, $P=0.001$ ), LDH $(P=0.029)$, and ferritin $(P=0.005)$, as shown in Figure 1. Sex was not significantly associated with $\mathrm{DC}(P=0.177)$, although a trend for more frequent DC in females (63\% vs $37 \%$ ) was observed.
Table I Patient characteristics

\begin{tabular}{|c|c|c|}
\hline $\begin{array}{l}\text { Age at start of } \\
\text { immunotherapy }\end{array}$ & $\begin{array}{l}\text { Median } \\
\text { Range }\end{array}$ & $\begin{array}{l}67.64 \\
43.68-84.26\end{array}$ \\
\hline Sex, n (\%) & $\begin{array}{l}\text { Female } \\
\text { Male }\end{array}$ & $\begin{array}{l}24(43) \\
32(57)\end{array}$ \\
\hline ECOG, n (\%) & $\begin{array}{l}0 \\
1 \\
2 \\
3\end{array}$ & $\begin{array}{l}18(32) \\
26(46) \\
8(14) \\
4(7)\end{array}$ \\
\hline Histology, n (\%) & $\begin{array}{l}\text { Adeno-Ca } \\
\text { SCC } \\
\text { Mixed } \\
\end{array}$ & $\begin{array}{l}4 I(73) \\
10(18) \\
5(9) \\
\end{array}$ \\
\hline PD-LI expression, $n(\%)$ & $\begin{array}{l}0 \% \\
\geq 1 \% \\
\text { Missing }\end{array}$ & $\begin{array}{l}14(25) \\
29(52) \\
13(23)\end{array}$ \\
\hline Tobacco (pack-years) & $\begin{array}{l}\text { Median } \\
\text { Range }\end{array}$ & $\begin{array}{l}47.5 \\
0-99\end{array}$ \\
\hline Metastatic sites, n (\%) & $\begin{array}{l} \\
2 \\
3 \\
4 \\
5 \\
6 \\
7 \\
\end{array}$ & $\begin{array}{l}3(5) \\
10(18) \\
11(20) \\
17(30) \\
8(14) \\
5(9) \\
2(4) \\
\end{array}$ \\
\hline $\begin{array}{l}\text { Type of immunotherapy, } \\
n(\%)\end{array}$ & $\begin{array}{l}\text { Atezolizumab } \\
\text { Nivolumab } \\
\text { Pembrolizumab }\end{array}$ & $\begin{array}{l}8(14) \\
29(52) \\
19(34)\end{array}$ \\
\hline $\begin{array}{l}\text { Immunotherapy first vs } \\
\text { second line, } \mathrm{n}(\%)\end{array}$ & $\begin{array}{l}\text { First line } \\
\text { Second line }\end{array}$ & $\begin{array}{l}11(20) \\
45(80)\end{array}$ \\
\hline $\begin{array}{l}\text { Driver mutations, } \\
\text { n (\%) } \\
\text { KRAS } \\
\text { EGFR } \\
\text { ALK }\end{array}$ & $\begin{array}{l}\text { Yes } \\
\text { No } \\
\text { Missing } \\
\text { Yes } \\
\text { No } \\
\text { Missing } \\
\text { Yes } \\
\text { No } \\
\text { Missing }\end{array}$ & $\begin{array}{l}24(43) \\
20(36) \\
12(2 \mathrm{I}) \\
4(7) \\
40(7 \mathrm{I}) \\
12(22) \\
0(0) \\
44(79) \\
12(2 \mathrm{I})\end{array}$ \\
\hline $\begin{array}{l}\text { Type of prior systemic } \\
\text { therapy, n (\%) }\end{array}$ & $\begin{array}{l}\text { Platinum-based therapy } \\
\text { EGFR tyrosine kinase } \\
\text { inhibitor }\end{array}$ & $\begin{array}{l}4 I(9 I) \\
4(9)\end{array}$ \\
\hline $\begin{array}{l}\text { Response to prior } \\
\text { systemic therapy, n (\%) }\end{array}$ & $\begin{array}{l}\text { DC } \\
\text { NDC }\end{array}$ & $\begin{array}{l}32(7 \mid) \\
13(29)\end{array}$ \\
\hline
\end{tabular}

Notes: Yes: tested, mutation found; No: tested, no mutation found; Missing: no information available.

Abbreviations: DC, disease control (complete response, partial response, stable disease); ECOG, Eastern Cooperative Oncology Group; EGFR, estimated glomerular filtration rate; NDC, no disease control; PD-LI, programmed death ligand I; SCC, squamous cell carcinoma.

ROC curve statistics for the four significant predictors are given in Table S1. The strongest associations were found for number of organs with metastases $(\mathrm{AUC}=0.743)$ and ferritin (AUC $=0.734$ ). Ferritin and the ECOG performance status had high sensitivity, whereas LDH had relatively high specificity with the chosen cut points. 

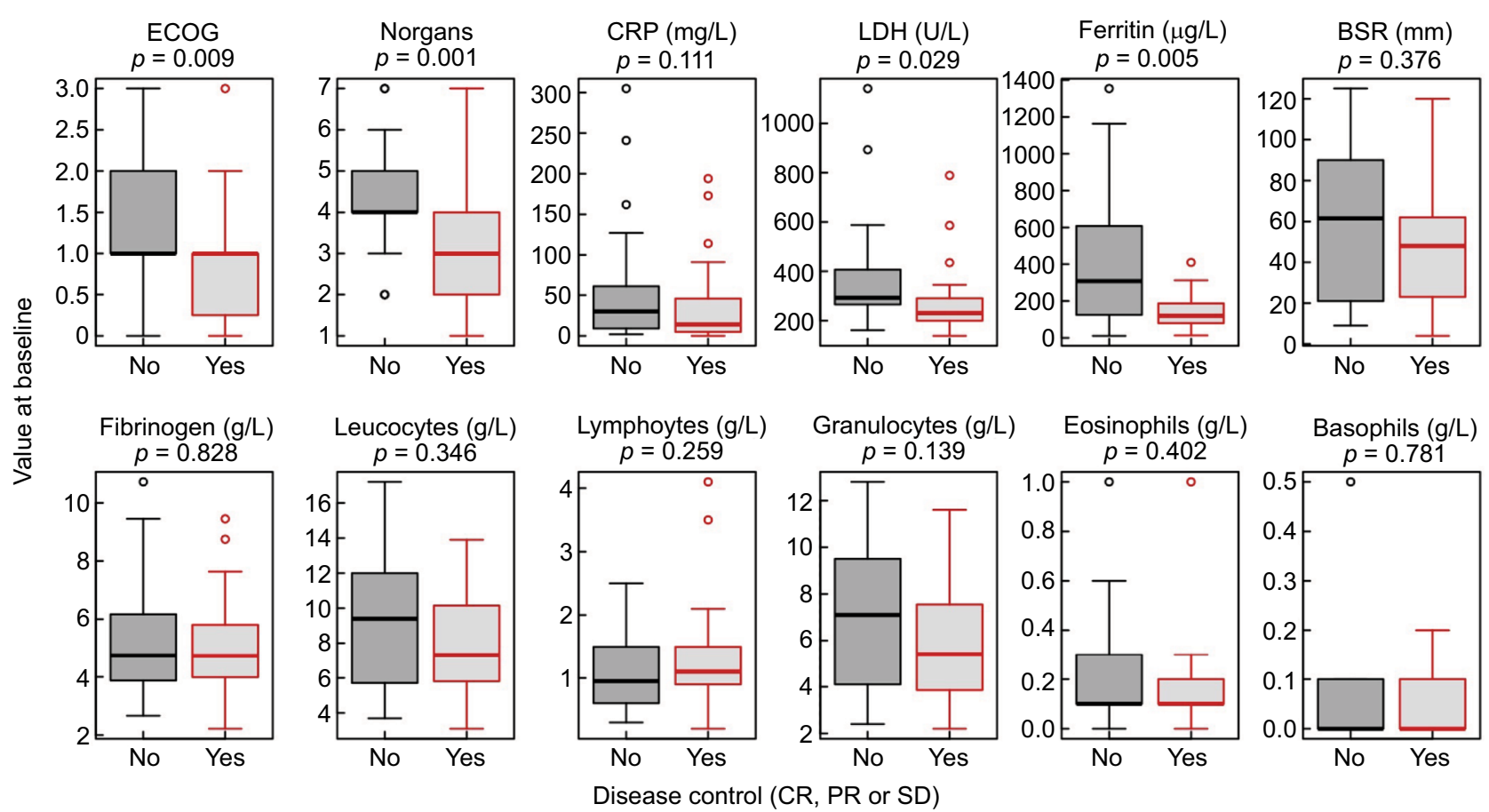

Figure I Distribution of baseline characteristics and laboratory parameters among patients with and without disease control.

Notes: Disease control was defined as either CR, PR, or SD. YES and NO means whether disease control was reached or not. The significance of differences between distributions (shifts in location) was determined with Wilcoxon rank sum tests.

Abbreviations: BSR, blood sedimentation rate; CR, complete remission; CRP, C-reactive protein; LDH, lactate-ehydrogenase; PR, partial remission; SD, stable disease.

Based on the ROC analysis, a risk score was created with the four identified parameters: ECOG performance status $\geq 2$, Norgans $\geq 4, \mathrm{LDH} \geq 262 \mathrm{U} / \mathrm{L}$, and ferritin $\geq 241 \mu \mathrm{g} / \mathrm{L}$. Patient numbers with each score value is shown in Table S1.

PD-L1 positivity $(\geq 1 \%)$ was not significantly different in the DC group vs NDC group $(P=0.826)$.

\section{Survival analyses}

Median follow-up time was 115 days (3.8 months). All patients with initial DC survived up to the end of follow-up, whereas most patients without DC died within 200 days (6.6 months). Both OS and PFS were significantly different between the DC and NDC groups $(P<0.001$, Figure 2$)$. Detailed rates for OS and PFS are shown in Table 2 with a CI of $95 \%$.

\section{Risk score}

A score ranging from 0 to 4 was created $(0=$ no risk factor, $4=$ four risk factors) based on the four factors identified. OS and PFS rates for patients grouped into the different score values are shown in Figure 3.

As scores 0 and 1 and scores 3 and 4 showed similar results, the following three categories were established: score $0-1$, score 2 , and score $3-4$. Survival analyses for the three different groups are shown in Figure 4 with detailed survival rates in Table 2.

\section{Discussion}

This prospective study identified risk factors for NSCLC patients undergoing therapy with a PD-1/PD-L1 antibody.

We identified ECOG performance status, number of organs with metastases, baseline LDH, and baseline ferritin as the most relevant independent factors for DC and survival in our cohort. Generally, the CR and PR rates in our cohort are in line with Phase III trials that led to the approval of the various antibodies. ${ }^{2-5}$

ECOG performance status and number of organs with metastases were already recognized as important adverse factors in the preimmunotherapy era that underlines their importance in daily practice. ${ }^{21-23}$ Furthermore, elevated baseline LDH is an established adverse factor in metastatic melanoma ${ }^{14-16,21}$ and its negative impact on survival was also recently shown for metastatic NSCLC. ${ }^{24}$

Inflammation is a hallmark driver for development and progression of malignancies. Additionally, cancer-related inflammation may be associated with tumor stage and prognosis. ${ }^{18}$ Several markers, such as CRP and neutrophil granulocytes, are associated with poor outcome in 

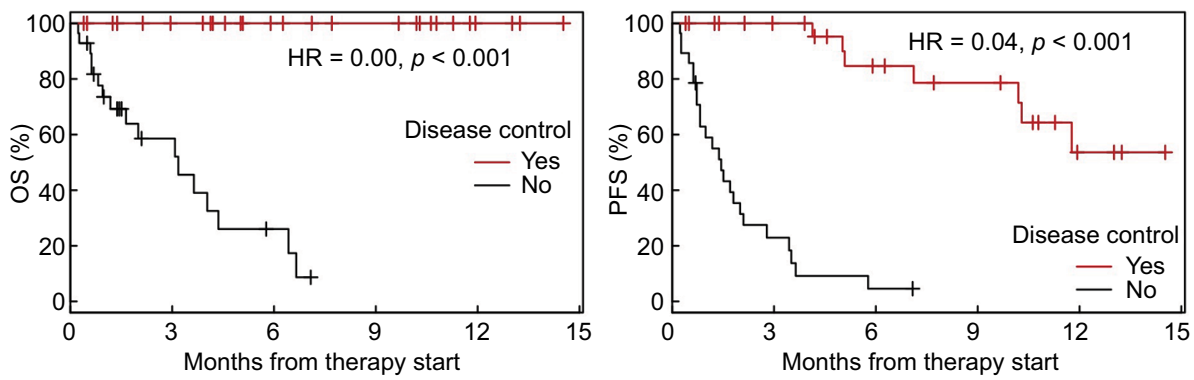

Figure 2 Kaplan-Meier curves showing OS and PFS for patients with or without DC at the first CT scan.

Notes: HRs for DC vs NDC are given together with $P$-values from log-rank tests. Crosses indicate censored observations.

Abbreviations: CT, computerizd tomography; DC, disease control, NDC, no disease control; OS, overall survival; PFS, progression-free survival.

Table 2 Comparison of 6 months and 12 months survival rates in the entire cohort, DC group and NDC group, and in the three different risk score groups

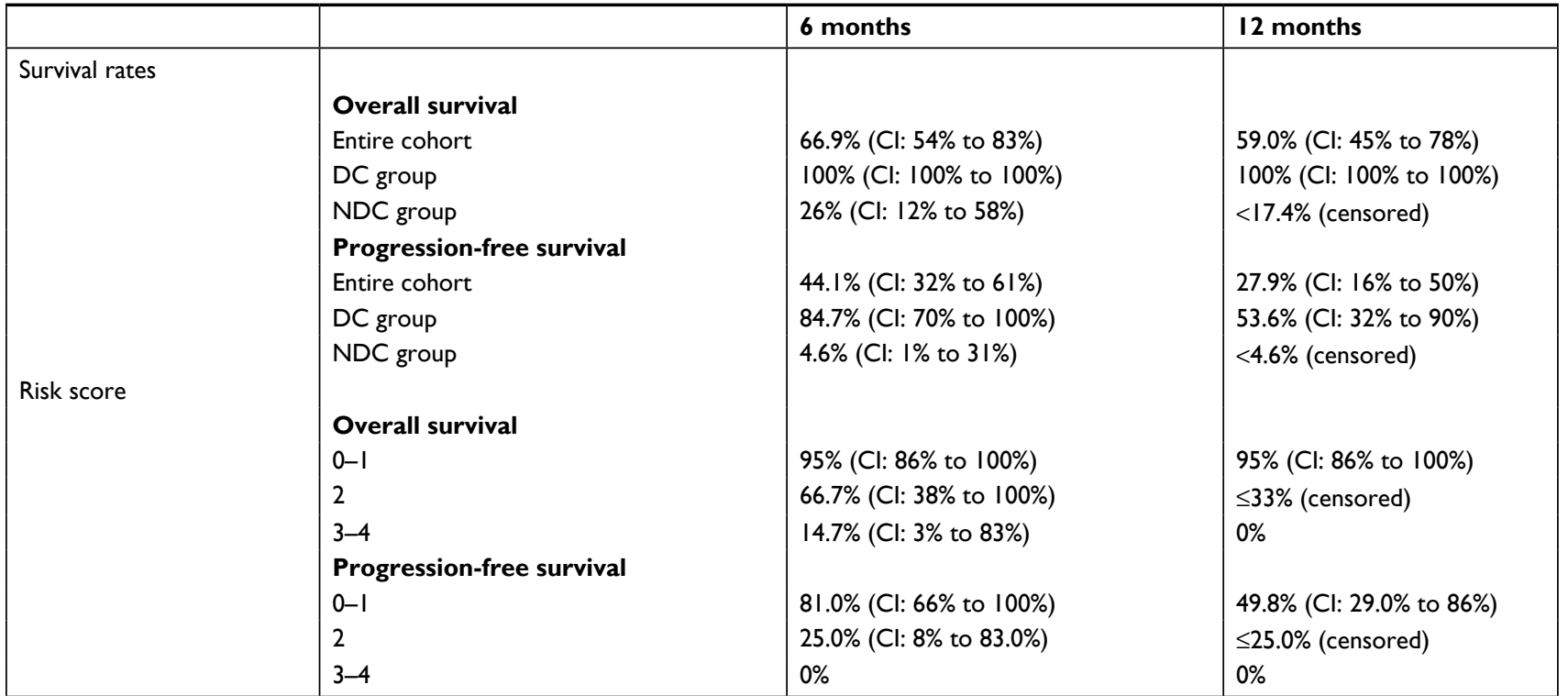

Abbreviations: DC, disease control; NDC, no disease control.
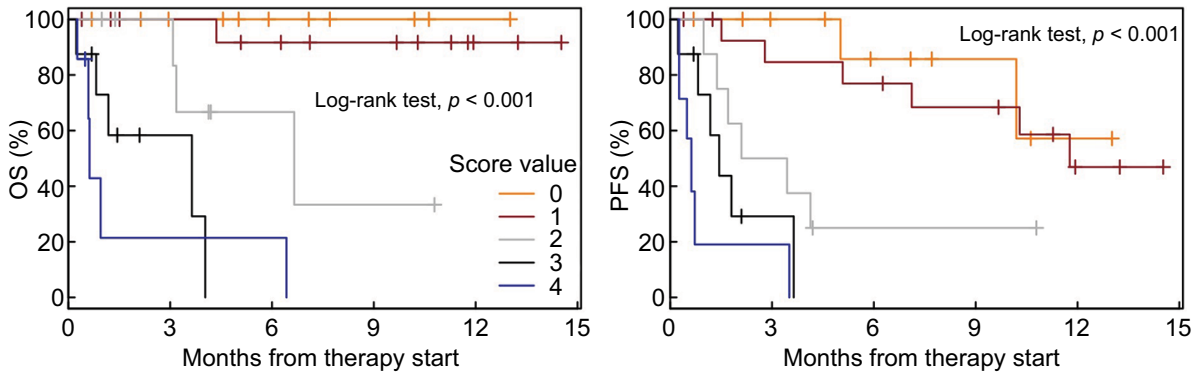

Figure 3 Kaplan-Meier curves showing OS and PFS for patients grouped by risk score values.

Abbreviations: OS, overall survival; PFS, progression-free survival.

melanoma and NSCLC patients treated with a checkpoint inhibitor. ${ }^{13,17,24-26}$ Apart from CRP, little is known about the relation between treatment outcome and other acute phase proteins, which are also usually elevated in inflammation processes. In our analysis ferritin is significantly associated with poor DC and survival. BSR, fibrinogen, and CRP show a trend, though not statistically significant. To our knowledge, our study is the first to evaluate the role of acute phase proteins in the context of checkpoint inhibitors in NSCLC patients. 

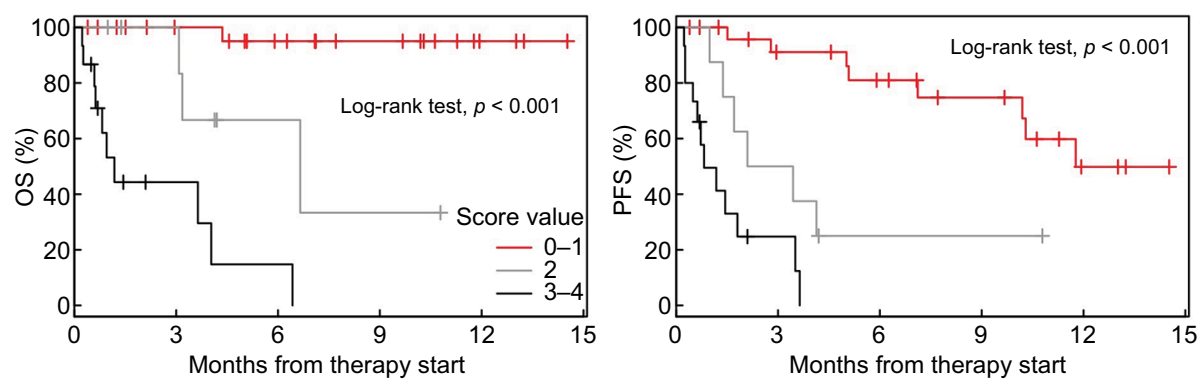

Figure 4 Kaplan-Meier curves showing OS and PFS for patients grouped into three groups by risk score.

Abbreviations: OS, overall survival; PFS, progression-free survival.

Based on the four risk factors identified we created a risk score that enables to stratify patients into different risk groups before treatment start. A similar risk score was recently published with a melanoma cohort being treated with the CTLA-4 antibody ipilimumab. ${ }^{16}$ In this study ECOG performance status, number of organs involved, and baseline LDH were identified as the most negative prognostic factors. When categorizing patients into different risk groups, OS significantly decreased with the number of risk factors before treatment initiation. ${ }^{16}$ Such a risk score can be easily performed in daily clinical routine. ECOG performance status is routinely done before treatment start, number of organs with metastases is determined by baseline imaging, and LDH and ferritin can also be routinely measured at baseline.

The following limitations of the study have to be addressed: First, the relatively small number of patients, which did not allow performing comprehensive multivariable analyses. However, our findings were statistically significant with clinical relevance for treating physicians. Second, it was not possible to validate our results with an external data set because several baseline blood values (eg, ferritin and BSR) are not routinely performed in daily practice. So, using a retrospective validation cohort is not meaningful. However, we believe that the study's prospective character strengthens our findings.

In summary, we hereby propose an easy-to-apply risk score that categorizes patients into different risk groups before treatment start with a PD-1/PD-L1 antibody. Our findings suggest that patients with a high score should be more closely monitored, and that these patients should be switched to another treatment rather early if lack of benefit is suspected. This will help to avoid unnecessary and potentially toxic treatments and side effects and to decrease treatment costs. In addition, patients may be offered alternative potentially active treatment options such as chemotherapy earlier, before a decline of performance status may preclude further systemic treatment. Although intriguing, our results have to be confirmed in larger prospective studies for validation.

\section{Acknowledgment}

This work was supported by the Swiss National Science Foundation (grant PP00P3_157448).

\section{Disclosure}

MF received research support from BMS and AstraZeneca (not for this project); Honoraria to institution for advisory: MSD, Roche, Astra, BMS, Boehringer. The authors report no other conflicts of interest in this work.

\section{References}

1. Moro-Sibilot D, Smit E, de Castro Carpeño J, et al. Outcomes and resource use of non-small cell lung cancer (NSCLC) patients treated with first-line platinum-based chemotherapy across Europe: FRAME prospective observational study. Lung Cancer. 2015;88(2):215-222.

2. Brahmer J, Reckamp KL, Baas P, et al. Nivolumab versus docetaxel in advanced squamous-cell non-small-cell lung cancer. $N$ Engl $J$ Med. 2015;373(2):123-135.

3. Borghaei H, Paz-Ares L, Horn L, et al. Nivolumab versus docetaxel in advanced nonsquamous non-small-cell lung cancer. $N$ Engl J Med. 2015;373(17):1627-1639.

4. Reck M, Rodríguez-Abreu D, KEYNOTE-024 Investigators, et al. Pembrolizumab versus chemotherapy for PD-L1-positive non-small-cell lung cancer. $N$ Engl J Med. 2016;375(19):1823-1833.

5. Rittmeyer A, Barlesi F, OAK Study Group, et al. Atezolizumab versus docetaxel in patients with previously treated non-small-cell lung cancer (OAK): a phase 3, open-label, multicentre randomised controlled trial. Lancet. 2017;389(10066):255-265.

6. Rizvi NA, Hellmann MD, Snyder A, et al. Cancer immunology. Mutational landscape determines sensitivity to PD-1 blockade in non-small cell lung cancer. Science. 2015;348(6230):124-128.

7. Peters S, Creelan BHM. Impact of tumor mutation burden on the efficacy of first-line nivolumab in stage iv or recurrent non-small cell lung cancer: an exploratory analysis of CheckMate 026. In: AACR Annual Meeting; April 1-5, 2017; Washington, DC. Abstract CT082.

8. Carbone DP, Reck M, CheckMate 026 Investigators, et al. First-line nivolumab in Stage IV or recurrent non-small-cell lung cancer. $N$ Engl J Med. 2017;376(25):2415-2426.

9. Skoulidis F, Hellmann MD, Awad MM, Rizvi H, Brett W, Carter WD. STK11/LKB1 co-mutations to predict for de novo resistance to PD-1/ PD-L1 axis blockade in KRAS-mutant lung adenocarcinoma. $J$ Clin Oncol. 2017;35(15 Suppl):9016. 
10. Zaretsky JM, Garcia-Diaz A, Shin DS, et al. Mutations associated with acquired resistance to PD-1 blockade in melanoma. $N$ Engl J Med. 2016;375(9):819-829.

11. Hellmann MD, Ciuleanu TE, Pluzanski A, et al. Nivolumab plus ipilimumab in lung cancer with a high tumor mutational burden. $N$ Engl J Med. 2018;378(22):2093-2104.

12. Delyon J, Mateus C, Lefeuvre D, et al. Experience in daily practice with ipilimumab for the treatment of patients with metastatic melanoma: an early increase in lymphocyte and eosinophil counts is associated with improved survival. Ann Oncol. 2013;24(6):1697-1703.

13. Ferrucci PF, Ascierto PA, Pigozzo J, et al. Baseline neutrophils and derived neutrophil-to-lymphocyte ratio: prognostic relevance in metastatic melanoma patients receiving ipilimumab. Ann Oncol. 2016;27(4):732-738.

14. Kelderman S, Heemskerk B, van Tinteren H, et al. Lactate dehydrogenase as a selection criterion for ipilimumab treatment in metastatic melanoma. Cancer Immunol Immunother. 2014;63(5):449-458.

15. Diem S, Kasenda B, Spain L, et al. Serum lactate dehydrogenase as an early marker for outcome in patients treated with anti-PD-1 therapy in metastatic melanoma. Br J Cancer. 2016;114(3):256-261.

16. Diem S, Kasenda B, Martin-Liberal J, et al. Prognostic score for patients with advanced melanoma treated with ipilimumab. Eur J Cancer. 2015;51(18):2785-2791.

17. Simeone E, Gentilcore G, Giannarelli D, et al. Immunological and biological changes during ipilimumab treatment and their potential correlation with clinical response and survival in patients with advanced melanoma. Cancer Immunol Immunother. 2014;63(7): 675-683.
18. Diakos CI, Charles KA, Mcmillan DC, Clarke SJ. Cancer-related inflammation and treatment effectiveness. Lancet Oncol. 2014;15(11):e493-e503.

19. Eisenhauer EA, Therasse P, Bogaerts J, et al. New response evaluation criteria in solid tumours: revised RECIST guideline (version 1.1). Eur $J$ Cancer. 2009;45(2):228-247.

20. Hopkins AM, Rowland A, Kichenadasse G, et al. Predicting response and toxicity to immune checkpoint inhibitors using routinely available blood and clinical markers. Br J Cancer. 2017;117(7):913-920.

21. Balch CM, Gershenwald JE, Soong SJ, et al. Final version of 2009 AJCC melanoma staging and classification. J Clin Oncol. 2009;27(36): 6199-6206.

22. Eton O, Legha SS, Moon TE, et al. Prognostic factors for survival of patients treated systemically for disseminated melanoma. J Clin Oncol. 1998;16(3):1103-1111.

23. Manola J, Atkins M, Ibrahim J, Kirkwood J. Prognostic factors in metastatic melanoma: a pooled analysis of Eastern Cooperative Oncology Group trials. J Clin Oncol. 2000;18(22):3782-3793.

24. Mezquita L, Auclin E, Ferrara R, et al. Association of the Lung Immune Prognostic Index with immune checkpoint inhibitor outcomes in patients with advanced non-small cell lung cancer. JAMA Oncol. 2018;4(3):351-357.

25. Diem S, Schmid S, Krapf M, et al. Neutrophil-to-lymphocyte ratio (NLR) and platelet-to-lymphocyte ratio (PLR) as prognostic markers in patients with non-small cell lung cancer (NSCLC) treated with nivolumab. Lung Cancer. 2017;111:176-181.

26. Bagley SJ, Kothari S, Aggarwal C, et al. Pretreatment neutrophil-tolymphocyte ratio as a marker of outcomes in nivolumab-treated patients with advanced non-small-cell lung cancer. Lung Cancer. 2017;106:1-7. 


\section{Supplementary material}

Table SI ROC curve statistics for the prediction of initial disease control by individual parameters

\begin{tabular}{|l|l|l|l|l|l|l|l|}
\hline & $\mathbf{n}$ & AUC & Cut point & Sensitivity & Specificity & PPV & NPV \\
\hline Ferritin & 49 & 0.734 & 241 & 0.885 & 0.609 & 0.719 & 0.824 \\
\hline LDH & 55 & 0.673 & 262 & 0.607 & 0.815 & 0.773 & 0.667 \\
\hline Norgans & 56 & 0.743 & 4 & 0.679 & 0.786 & 0.760 & 0.710 \\
\hline ECOG & 56 & 0.696 & 2 & 0.929 & 0.357 & 0.591 & 0.837 \\
\hline
\end{tabular}

Abbreviations: AUC, area under the curve; cut point, maximizing the sum of sensitivity and specificity (DC is predicted by parameter values below the cut point); ECOG, Eastern Cooperative Oncology Group; LDH, lactate dehydrogenase; Norgans, number of involved organs; $n$, number of nonmissing observations; NPV, negative predictive value (initial PD rate among patients with values above the cut point); PPV, positive predictive value (initial DC rate among patients with values below the cut point).

\section{Publish your work in this journal}

Cancer Management and Research is an international, peer-reviewed open access journal focusing on cancer research and the optimal use of preventative and integrated treatment interventions to achieve improved outcomes, enhanced survival and quality of life for the cancer patient. The manuscript management system is completely online and includes a very quick and fair peer-review system, which is all easy to use. Visit http://www.dovepress.com/testimonials.php to read real quotes from published authors. 